\title{
Du traitement du signal avec des ondes et des lentilles : Le traitement d'images et la transformée de Fourier à la lumière de la diffraction en Optique
}

\author{
Jean-Claude Pissondes \\ jc.pissondes@iut-velizy.uvsq.fr \\ Adresse : IUT de Vélizy, Département Réseaux et Télécoms \\ 10-12 Avenue de l'Europe, 78140 Vélizy
}

\begin{abstract}
RESUME : On présente, dans cette contribution, les trois volets du travail que des étudiants de deuxième année en DUT Réseaux et Télécoms doivent réaliser dans le cadre d'un projet tuteuré. Dans un premier temps, on rappellera le rôle bien particulier de la transformée de Fourier dans la diffraction de Fraunhofer : dans un tel cadre, les propriétés de la transformée de Fourier, et la transformée elle-même, se localisent dans l'espace physique et se visualisent. Dans un second temps, on décrira l'expérience d'Abbe où est mise en évidence la possibilité d'opérer des modifications sur une image par filtrage des fréquences spatiales. On insistera sur le fait que ces opérations de filtrage, qui ont lieu au niveau du spectre de l'image, sont localisées dans l'espace : elles correspondent à des actions bien réelles d'obturation, de découpe ou de cache, situées spatialement dans le montage d'Abbe. Enfin, à l'aide du logiciel Matlab®, on utilisera une image témoin constituée de motifs simples pour voir les altérations qu'elle subit lors d'un processus de filtrage et d'échantillonnage. Ce projet vise à sortir la transformée de Fourier du contexte du traitement du signal et à en éclairer les aspects les plus connus. Ainsi, les étudiants observent en les voyant des propriétés qu'ils auront étudiées dans leur cursus. L'intérêt pédagogique d'un tel travail est la prise de recul sur des notions et opérations vues dans un cadre spécifique. Une telle approche, privilégiant les allers et retours entre deux domaines distincts pour éclairer un même concept, a bien sûr vocation à être étendue.
\end{abstract}

Mots clés : Transformée de Fourier, Optique, diffraction, filtrage des fréquences spatiales, traitement du signal

\section{INTRODUCTION}

\subsection{Considérations générales}

L'apprentissage d'un outil mathématique appliqué de façon exclusive à une discipline donnée, fait perdre de vue ce qu'il a d'universel. Par exemple, l'enseignement des Réseaux et des Télécommunications en IUT requiert l'initiation au traitement du signal, et plus généralement à l'analyse de Fourier. Ces enseignements conduisent souvent les étudiants, comme leurs enseignants, à associer la transformée de Fourier à la dualité temps-fréquence exclusivement. Pourtant, bien avant la théorie du signal, les premiers calculs de transformée de Fourier concernent des calculs de figures de diffraction associés à une «correspondance espace-espace »: ouverture diffractante et figure de diffraction sont reliées par transformée de Fourier, mais sont localisées dans l'espace réel. De plus, les exemples où les variables conjuguées sont des coordonnées spatiales et des fréquences spatiales se rencontrent souvent en Physique et apparaissent, pour certains, avant la théorie du signal.

Les caractéristiques fréquentielles d'un ensemble de mesures - c'est-à-dire d'un « signal » -, mises en évidence grâce à sa représentation fréquentielle, sont bien réelles mais ne correspondent à rien qui soit directement visible. En traitement du signal, le passage dans le « domaine fréquentiel » correspond à l'observation d'effets et de caractéristiques qui n'ont de réel que leur contrepartie temporelle [11].

Le but affiché du projet tuteuré décrit ici, est donc d'éclairer certains aspects de la transformée de Fourier par son utilisation dans des disciplines très différentes et a priori étrangères : on utilise ce qui est évident et visible dans un domaine - en l'occurrence l'Optique pour mieux concevoir ce qui s'appréhende de façon indirecte, voire abstraite, en traitement du signal [12].

\subsection{Omniprésence de l'analyse de Fourier}

Enfin, on sensibilisera les étudiants, tout au long du projet, au caractère universel et à l'omniprésence de l'analyse de Fourier en Physique. Une présence mathématique d'abord, dans la résolution des équations différentielles qui expriment les lois de la Physique, en particulier dans le cas où l'on bénéficie de la linéarité, dont les solutions se développent en «somme » de Fourier, série ou intégrale. Une présence d'un point de vue physique ensuite, puisque les grandeurs qui se propagent ou se comportent comme une onde sont partout présentes, en Mécanique et en acoustique, en électromagnétisme et en Optique, mais aussi dans la Physique contemporaine: mécanique quantique et relativité générale. Dès lors, des décompositions en somme de Fourier, discrète ou continue, apparaissent naturellement dans la représentation du phénomène décrit [2],[3],[4].

\section{DIFFRACTION ET TRANSFORMEE DE FOURIER}

\subsection{Description ondulatoire de la lumière}

Le cas de l'Optique ondulatoire est particulièrement éloquent puisqu'il donne à voir ce qui, ailleurs, devra seulement se concevoir, en particulier dans le cadre de l'analyse de Fourier. Mais pour cela, il faut disposer 
d'une théorie, et donc d'équations, qui rendent compte du comportement de la lumière, au moins pour certaines de ses propriétés. Parmi les grands promoteurs d'une théorie ondulatoire de la lumière, c'est Fresnel qui, le premier, saura mettre clairement en évidence les caractéristiques ondulatoires de la lumière. Il étendra le principe de Huygens décrivant une propagation «de proche en proche » et concevra des méthodes graphiques et calculatoires pour déterminer l'amplitude en un point de l'espace (sur un écran, par exemple) de la lumière diffractée par un obstacle ou une « ouverture ». Critiqués par Kirchhoff, certains aspects de ses calculs manqueront de généralité, voire de rigueur [1]. Ce dernier proposera alors une intégrale illustrant mathématiquement le principe de Huygens-Fresnel de façon très générale. Indiquons juste que Kirchhoff montre qu'une amplitude $A$, solution de l'équation de Helmholtz, fait l'objet du théorème suivant: «en tout point $P$ à l'intérieur d'une surface fermée $S$, l'amplitude $A(P)$ se déduit des valeurs de $A_{S}$ et de ses dérivées partielles $s u r$ la surface $S \gg($ fig 1$)$. Plus précisément, l'amplitude $A(P)$ s'exprime comme une intégrale double où interviennent $A_{S}$ et ses dérivées partielles. L'intégrale de Kirchhoff, plus riche et plus générale que les formules de Fresnel, est difficilement utilisable telle quelle. Néanmoins, elle autorise, suite à certaines approximations, des calculs conformes à l'expérience et redonne les expressions introduites par Fresnel dont la justification est parfois difficile [4], [6], [7].

\subsection{De Kirchhoff à Fresnel}

Le schéma fig 1 donne la configuration spatiale dans le cas où un diaphragme (l'ouverture au centre du schéma) fait partie de la surface $S$. Une source $P_{0}$ émet une onde sphérique de la forme $\left(A_{0} / r\right) \exp (i k r)$, où $k=2 \pi / \lambda$.

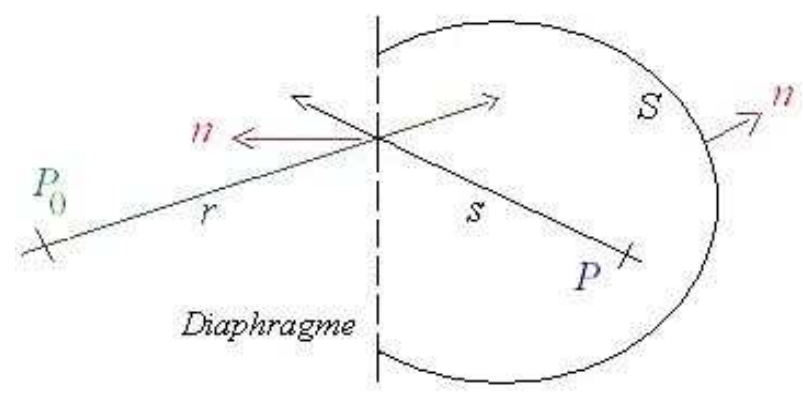

fig 1 : Amplitude en $P$, après émission à partir de $P_{0}$

Dans le cas où les distances $r$ et $s$ sont «grandes » par rapport à l'ouverture du diaphragme, elle-même «très grande » par rapport à la longueur d'onde $\lambda$, on montre que l'intégrale de Kirchhoff devient

$$
A(P)=\frac{-i A_{0}}{2 \lambda} \iint_{\text {Diaph }} \frac{e^{i k(r+s)}}{r s}[\cos (\vec{n}, \vec{s})-\cos (\vec{n}, \vec{r})] d \sigma
$$

où la contribution à l'intégrale de surface hors du diaphragme est considérée comme nulle. Considérons à présent le point $P$ situé sur un écran placé à une distance $z$ du diaphragme (fig 2). Notons $(x, y)$ les coordonnées de $M$ sur le diaphragme, et $(u, v)$ celles de $P$.

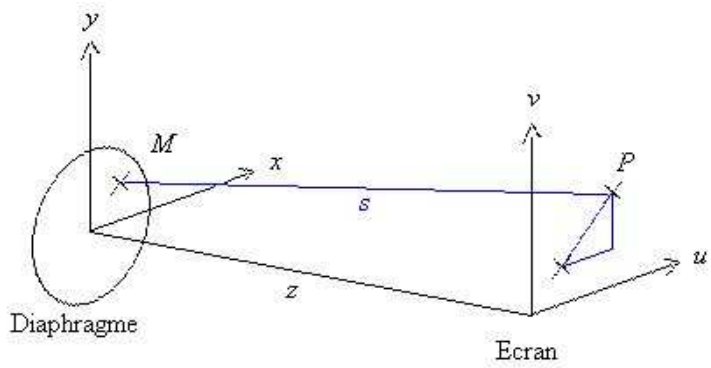

fig 2 : De l'ouverture à l'écran

En utilisant le théorème de Pythagore reliant $s$ et $z$, en supposant que les dimensions de l'écran et du diaphragme soient petites par rapport à $z$ et en ne considérant que les rayons quasiment parallèles à l'axe $\mathrm{O} z$, on vérifie que (1) se réécrit comme

$$
A(P)=\frac{-i e^{i k z}}{\lambda z} \iint_{\text {Diaph }} A(M) e^{i \pi\left[\frac{(u-x)^{2}+(v-y)^{2}}{\lambda z}\right]} d x d y
$$

La relation (2) donne, dans l'approximation de Fresnel, l'amplitude $A(P)=A(u, v)$ du champ sur l'écran à partir de toutes les contributions $A(M)=A(x, y)$ sommées au niveau du diaphragme, en fonction des paramètres du problème (géométrie et longueur d'onde). En considérant le carré $d u$ module de $A(P)$, on obtient ce que le «détecteur» œil capte : une figure de diffraction, ici dans l'approximation de Fresnel, correspondant à la répartition sur l'écran de l'intensité lumineuse après le passage de la lumière par le diaphragme.

\subsection{De Fresnel à Fraunhofer}

Le dernier niveau d'approximation consiste à éloigner davantage l'écran du diaphragme (on ne discutera pas des distances possibles entre $P_{0}$ et le diaphragme). On a alors $z>\left(x^{2}+y^{2}\right) / \lambda$ tout en considérant que les produits $u x$ et $v y$ l'emportent sur les termes quadratiques $x^{2}$ et $y^{2}$. Les distances $s$ et $z$ sont maintenant reliées par

$$
s \approx z+\left(u^{2}+v^{2}\right) / 2 z-(u x+v y) / z
$$

En intégrant sur $\mathbb{R}^{2}$, l'amplitude écrite en (2) devient

$$
A(u, v)=\frac{-i e^{i k z}}{\lambda z} e^{i \pi\left(\frac{u^{2}+v^{2}}{\lambda z}\right)} \iint A(x, y) e^{-2 i \pi\left(\frac{u x+v y}{\lambda z}\right)} d x d y
$$

On reconnaît dans l'intégrale (4), la transformée de Fourier bidimensionnelle au point $(u / \lambda z, v / \lambda z)$ de l'amplitude $A(x, y)$. Par conséquent, l'amplitude $d u$ champ associée à la diffraction par une ouverture, dans le cadre de l'approximation de Fraunhofer, est proportionnelle à la transformée de Fourier de l'amplitude du champ au niveau du diaphragme. Ayant supposé une distance très grande entre le diaphragme et l'écran, on parle de diffraction à l'infini.

On introduit alors les fréquences spatiales

$$
p_{x}=u / \lambda z \text { et } p_{y}=v / \lambda z \text {. }
$$

Ces grandeurs ont pour dimension l'inverse d'une longueur et jouent dans la suite le rôle que joue la fréquence temporelle en traitement du signal. La figure de diffraction visualisée sur l'écran correspond à l'intensité lumineuse vue par l'œil, c'est-à-dire au carré 
du module de $A(u, v)$. Dans l'approximation de Fraunhofer, elle est donc donnée par

$$
I\left(p_{x}, p_{y}\right)=I_{0}\left|\hat{A}\left(p_{x}, p_{y}\right)\right|^{2}
$$

où $\hat{A}$ désigne la transformée de Fourier de A. Chaque point du spectre d'amplitude (au carré) sur l'écran est donc repéré par les coordonnées fréquentielles $\left(p_{x}, p_{y}\right)$. Les basses fréquences sont alors situées au centre de l'écran, et les hautes fréquences... loin du centre.

Remarquons que, selon (6), et d'après ce que l'on constate expérimentalement, la figure de diffraction dans l'approximation de Fraunhofer est invariante par translation dans le plan $(x, y)$ du diaphragme. En effet, le facteur de phase apparaissant du fait de la translation, disparaît dans l'écriture du module.

Un montage optique permettant de «simuler» la diffraction à l'infini utilise deux lentilles convergentes $L$ de part et d'autre du diaphragme (fig 3): des rayons parallèles arrivent sur l'ouverture (une fente verticale), et les rayons parallèles associés à la diffraction arrivent «de l'infini » sur l'écran placé au niveau du «plan image $»$ de la seconde lentille.

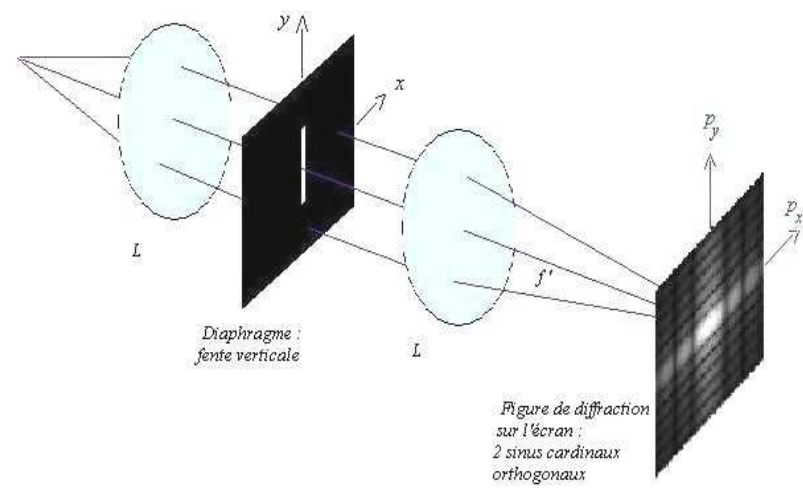

fig 3 : Diffraction à l'infini

\subsection{Quelques exemples}

\subsubsection{Que s'attend-on à voir ?}

Nous savons à présent quels sont les «motifs » de diffraction que nous observerons dans l'approximation de Fraunhofer : connaissant la fonction bidimensionnelle associée à l'ouverture diffractante, en particulier à sa géométrie, sa transformée de Fourier (son module au carré) nous donnera la figure de diffraction attendue. La figure qui suit (fig 4) rassemble les principaux spectres bien connus des praticiens (enseignants et étudiants) du traitement du signal. Se rapportant à la dualité temps-fréquence, ils relient deux «fonctions », toutes deux à une variable. Leur utilisation et une combinaison judicieuse de ces graphes nous donneront facilement la fonction de deux variables spatiales associée à la figure de diffraction cherchée, pour une ouverture diffractante donnée. Mais, le cheminement inverse est au moins aussi intéressant, celui que pratique le physicien, le cristallographe ou le biologiste : observant une figure de diffraction, et ayant bien à l'esprit nos spec- tres de base, on remonte à la géométrie de l'obstacle diffractant (agencement d'atomes dans un cristal, structure d'un fragment d'ADN, etc.).

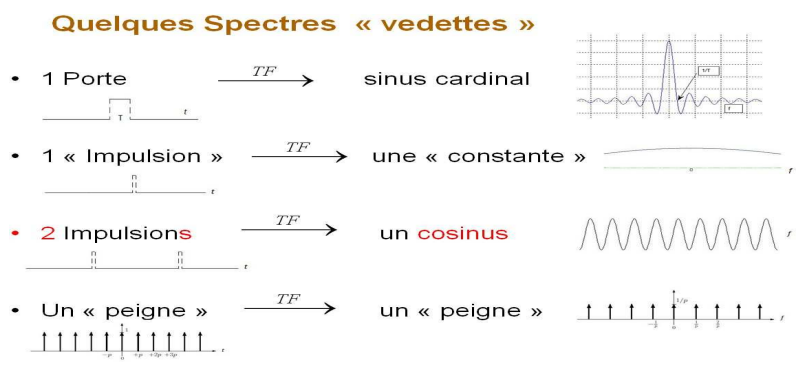

fig 4 : Principaux spectres de base en traitement du signal

\subsubsection{Ouverture rectangulaire}

On considère ici que l'ouverture diffractante est une fente de hauteur $a$ et de largeur $b$, découpée dans un cache opaque de dimensions très grandes par rapport à $a$ et $b$. La fonction bidimensionnelle qui la représente est donc une "porte » à deux dimensions caractérisée par deux largeurs $a$ et $b$ selon deux axes orthogonaux. Si l'écran où l'on observe la diffraction est placé à une distance $z=D$ suffisamment grande de la fente, nous sommes dans le cadre de l'approximation de Fraunhofer. L'amplitude de la lumière diffractée est alors donnée par la transformée de Fourier à deux dimensions de la fonction associée à la fente. L'intensité associée à la figure de diffraction observée, est donc proportionnelle au carré de deux sinus cardinaux :

$$
I\left(p_{x}, p_{y}\right)=I_{0}[\sin (\alpha) / \alpha]^{2}[\sin (\beta) / \beta]^{2},
$$

où $\alpha=\pi a p_{x}=\pi a u / \lambda D$ et $\beta=\pi b p_{y}=\pi b v / \lambda D$. Pour une longueur d'onde $\lambda$ donnée, elle s'annule une première fois de part et d'autre du centre pour $p_{x}= \pm 1 / a$ et $p_{y}=$ $\pm 1 / b$.
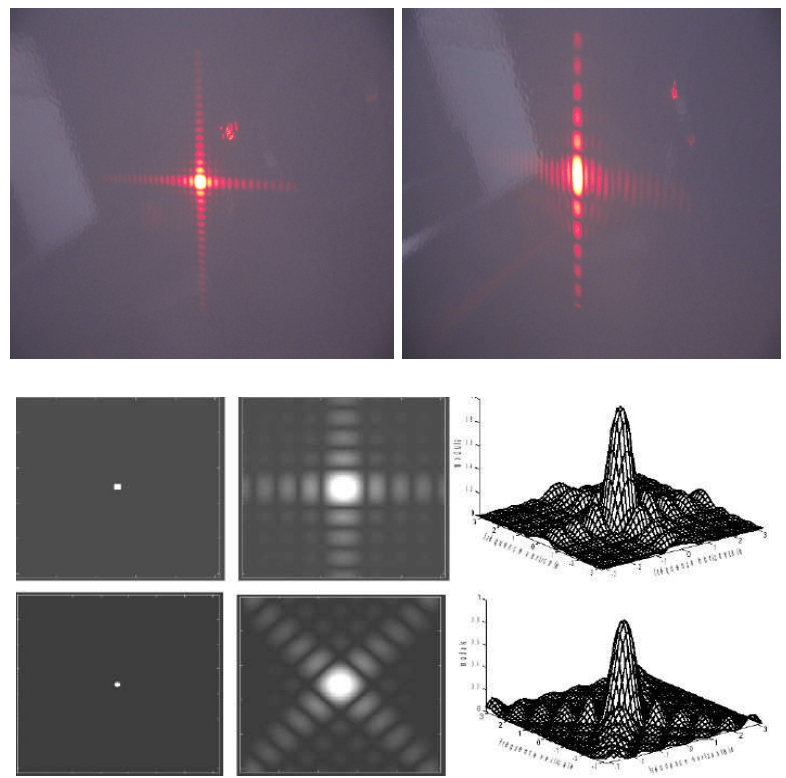

fig 5 : Diffraction de Fraunhofer par une ouverture carrée et par une fente. Effet d'une rotation 
L'allure de ce que l'on voit sur l'écran dépend des paramètres géométriques $D, a$, et $b$ : comme on s'y attend, plus la fente est étroite, plus la figure de diffraction est dilatée orthogonalement à la fente (fig 3 et fig 5). Une rotation dans le plan $(x, y)$ induit une rotation de même angle dans le plan $\left(p_{x}, p_{y}\right)$ (fig 5 en bas).
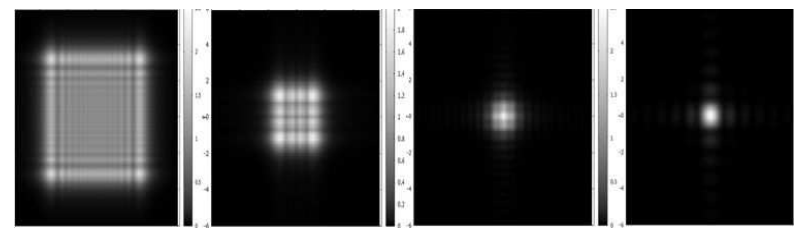

fig 6 : De Fresnel à Fraunhofer

Enfin, sachant ce que l'on obtient avec l'ouverture rectangulaire, observons la fig 6 où, de gauche à droite, on présente sa figure de diffraction lorsque l'écran où elle se forme s'éloigne de l'ouverture. On passe alors de l'approximation de Fresnel à l'approximation de Fraunhofer, où l'on reconnaît clairement, à droite, les sinus cardinaux attendus.

\subsubsection{Diffraction et interférences par 2 ouvertures}

L'expérience des trous (ou fentes) d'Young est bien connue : une onde unique passe par deux petites ouvertures écartées d'une distance $L$. Les trous se comportent comme deux sources cohérentes qui donnent lieu à des interférences, après diffraction de l'onde incidente par chaque trou. Ce que l'on voit sur un écran placé à une distance $D$, grande devant $L$, est montré fig 7 : des « franges » d'interférences, modulées par des anneaux concentriques associés à la diffraction par chaque trou. Sur l'écran, deux franges sont séparées par la distance $\lambda D / L$. En Optique ondulatoire, comme en traitement du signal, la périodicité en $1 / L$ - ou en $1 / T$ - du motif spectral doit nous apparaître comme naturelle.
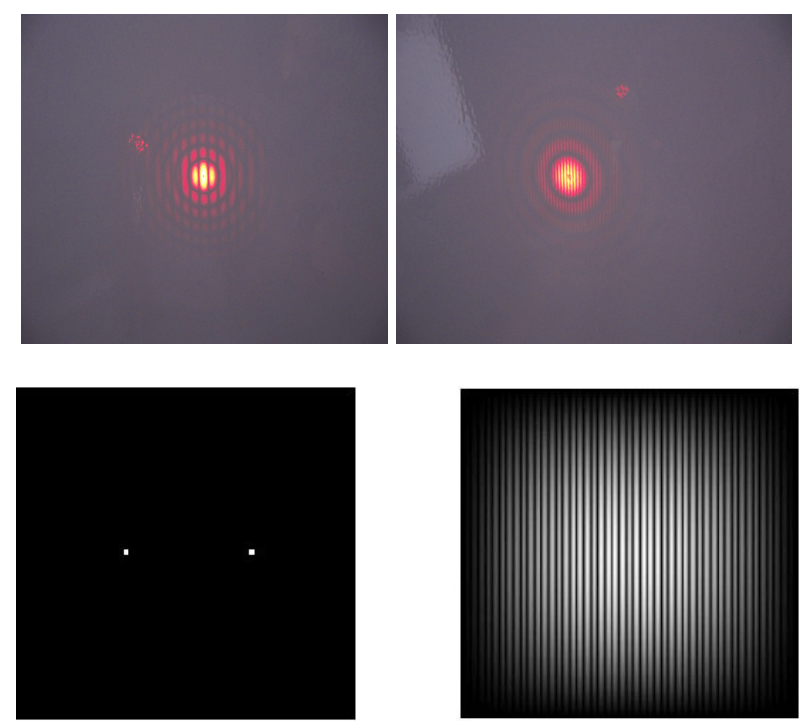

fig 7 : Diffraction et interférences par 2 trous : phénomène réel pour 2 valeurs de L et simulation
Notons bien que les deux trous sont nécessaires pour obtenir des interférences et le motif oscillant en cosinus correspondant (fig 7 à droite). Supprimer l'un des trous, supprime les interférences et l'oscillation associée. Ne reste alors que la diffraction par le trou restant. De même, il faut deux portes, ou impulsions, séparées dans le temps pour obtenir des oscillations en cosinus dans le spectre d'amplitude (voir le troisième spectre fig 4). La suppression de l'une des portes supprime ce terme, et ne laisse plus dans le spectre d'amplitude que le sinus cardinal associé à la porte restante.

\subsubsection{Diffraction et interférences par $N$ fentes}

Le cas d'un ensemble de fentes est intéressant parce qu'il conduit à la visualisation de la transformée de Fourier d'une fonction se rapprochant d'un «peigne de Dirac », moyennant les passages à la limite de deux paramètres: le nombre de fentes et la largeur de chaque fente. Considérons $N$ fentes de largeur $a$, de hauteur très grande devant $a$, et séparées deux à deux par une distance $L$. Dans les conditions de Fraunhofer, on obtient après un calcul de transformée de Fourier (dans la seule direction $x$ ), une intensité proportionnelle à un produit de deux termes :

$$
I=I_{0}[\sin (\alpha) / \alpha]^{2}[\sin (N \beta) / N \sin \beta]^{2}
$$

avec $\alpha=\pi a p_{x}$ et $\beta=\pi L p_{x}$.

L'intensité observée sur l'écran comprend donc: un «terme de diffraction» qui correspond à la diffraction par chaque fente : c'est le sinus cardinal au carré qui joue le rôle d'enveloppe des raies vertes fig 8 ; un «terme d'interférences » qui correspond aux interférences de la lumière issue des $N$ sources cohérentes que constituent les fentes : c'est l'ensemble des raies insérées dans le sinus cardinal.
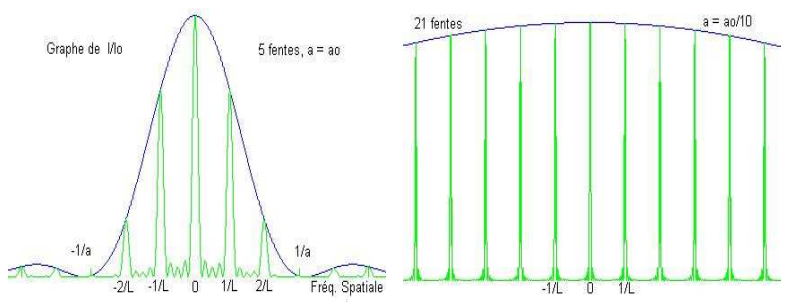

\section{fig 8 : Diffraction et interférences par $N$ fentes}

Quand $N$ augmente, chaque raie (de largeur en $1 / N$ ) est de plus en plus piquée, deux raies consécutives étant séparées par la même distance fréquentielle $1 / L$. Lorsque la largeur $a$ des fentes tend vers zéro, l'enveloppe que forme le sinus cardinal tend vers une droite horizontale. On voit, suite à cette double limite, se profiler l'allure d'un peigne de Dirac dans le domaine des fréquences spatiales (fig 8 à droite), alors même que les deux passages à la limite conduisent à un peigne dans le domaine spatial (remplacer la «porte» de largeur $a$ et de hauteur fixée, associée à la fente, par 1 '« impulsion » de largeur $a$ et de hauteur $1 / a$ ). 

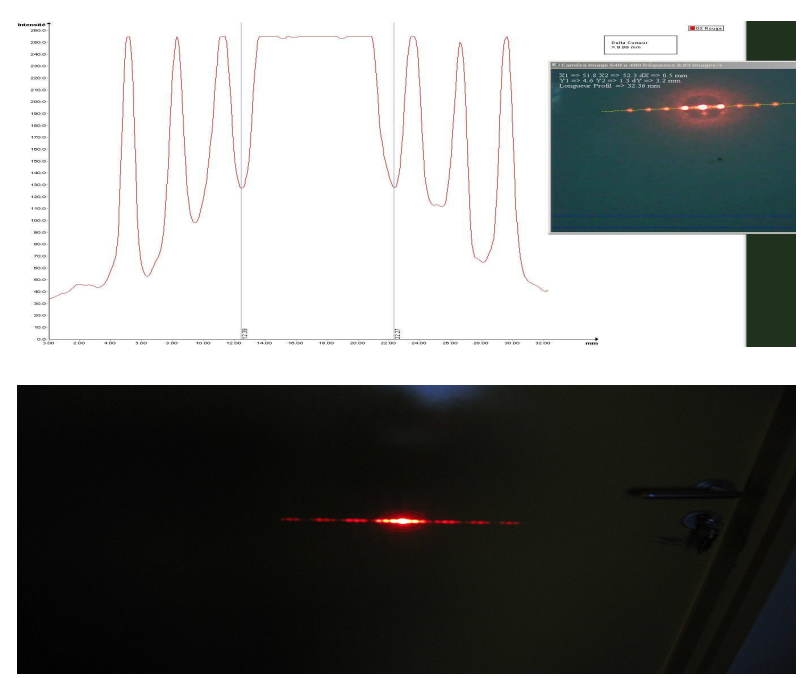

fig 9: Diffraction et interférences par $N$ fentes, en vrai

On reconnait sur la fig 9 la figure de diffraction par $N$ fentes : on voit clairement les raies associées au terme d'interférences « enveloppées » par le terme de diffraction en forme de sinus cardinal au carré.
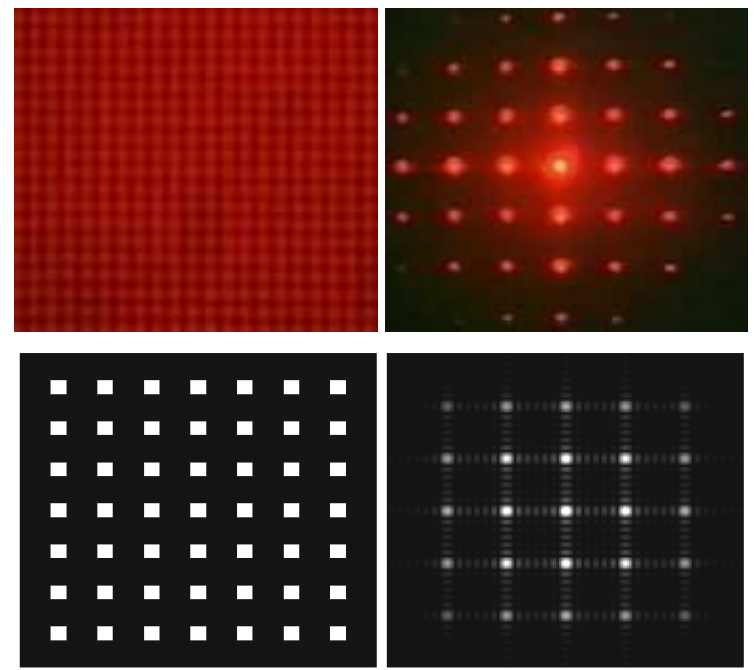

fig 10 : «Grille » et sa figure de diffraction-interférences : cliché et simulation

Enfin, on observe fig 10 une «grille » et son spectre. Ce dernier (la figure de diffraction-interférences associée) se déduit aisément des spectres fig 8 ou fig 9 , en considérant un ensemble d'alignements parallèles d'ouvertures carrées.

\section{L'EXPERIENCE D'ABBE}

\subsection{Description du montage}

L'expérience d'Abbe repose sur les caractéristiques de la diffraction à l'infini et l'obtention des figures de diffraction par transformée de Fourier. Cette expérience met clairement en évidence la possibilité d'opérer des modifications sur une image en travaillant sur son spectre. Elle correspond au montage suivant. On fixe à l'extrémité d'un banc optique une ouverture dans la- quelle on place une image translucide $\operatorname{Im}(x, y)$ (par exemple une diapositive, placée fig 11 à gauche). Celleci est éclairée par des rayons lumineux arrivant parallèlement sur l'image (grâce à une lentille non représentée). Pour simuler les conditions de Fraunhofer, on place (revoir fig 3) une lentille convergente $\mathrm{L}_{1}$ sur le chemin des rayons de la lumière diffractée par l'image $\operatorname{Im}(x, y)$, positionnée dans le plan objet de $\mathrm{L}_{1}$. La lentille ramène au niveau de son plan image tous les rayons parallèles qui auraient dû «se rencontrer à l'infini ». Ainsi, la figure de diffraction calculable par transformée de Fourier est ramenée par $\mathrm{L}_{1}$ à distance finie. Elle correspond au spectre de $\operatorname{Im}(x, y)$ et se trouve dans le plan image de $\mathrm{L}_{1}$ (fig 11 au centre en rouge) qu'on appelle donc plan de Fourier de la lentille. Notons-le $\mathrm{PF}_{1}$. Le montage d'Abbe comprend une seconde partie où le même processus optique se répète avec une seconde lentille identique $\mathrm{L}_{2}$ (partie droite de la fig 11). C'est le spectre de $\operatorname{Im}(x, y)$, situé au niveau du plan objet de $L_{2}$, qui joue alors le rôle de l' «image ». Les deux lentilles étant identiques et de même distance focale $f$, on parle de montage $4 f$. Dans le plan image, ou plan de Fourier $\mathrm{PF}_{2}$ de $\mathrm{L}_{2}$, on visualise donc la figure de diffraction à l'infini du spectre de $\operatorname{Im}(x, y)$, c'est-à-dire le spectre... de son spectre.

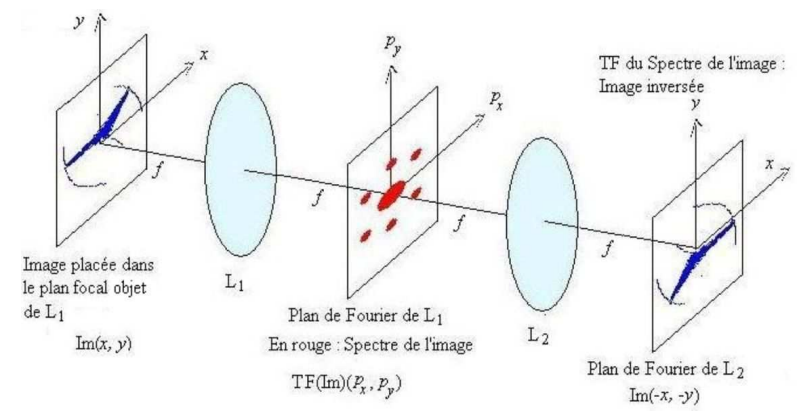

fig 11 : Expérience d'Abbe ou Montage $4 f$

Autrement dit, on observe dans le plan image de $\mathrm{L}_{2} l a$ double transformée de Fourier de l'image $\operatorname{Im}(x, y)$. Comme dans le cas unidimensionnel, la double transformée de Fourier d'un motif à deux dimensions, donne le motif lui-même, mais inversé par rapport à l'origine :

$$
[T F \circ T F](\operatorname{Im})(x, y)=\operatorname{Im}(-x,-y)
$$

On observe donc en $\mathrm{PF}_{2}$ l'image placée au niveau de l'ouverture éclairée, avant $\mathrm{L}_{1}$, mais inversée (fig 11 à droite).

\subsection{Ce que l'on fait réellement}

$\mathrm{Si}$ l'on récupère bien au niveau du plan de Fourier $\mathrm{PF}_{2}$ de $\mathrm{L}_{2}$ l'image placée dans le plan objet de $\mathrm{L}_{1}$, mais inversée, toute modification effectuée au niveau de son spectre en $\mathrm{PF}_{1}$ aura une incidence sur le contenu de l'image visualisée en $\mathrm{PF}_{2}$. Ainsi, il est possible de voir en $\mathrm{PF}_{2}$ les altérations de l'image qui font suite à la suppression volontaire ou accidentelle de certaines fréquences de son spectre en $\mathrm{PF}_{1}$. Si l'on supprime l'ensemble des fréquences situées à l'extérieur d'un 
disque placé en $\mathrm{PF}_{1}$, on réalise un filtrage passe-bas qui provoque l'adoucissement des contours visualisé fig 12, ainsi qu'une suppression des détails les plus fins.

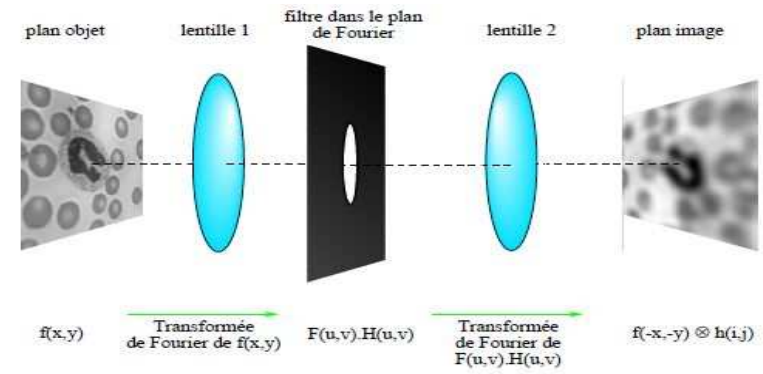

fig 12 : Filtrage passe-bas et suppression des détails

Inversement, placer un cache circulaire au centre du plan de Fourier $\mathrm{PF}_{1}$ supprime les basses fréquences : on effectue alors un filtrage passe-haut. Cela a pour conséquence d'accentuer les contours de l'image tout en atténuant les différences d'intensités entre les plages continues (fig 13). On réalise alors une strioscopie.

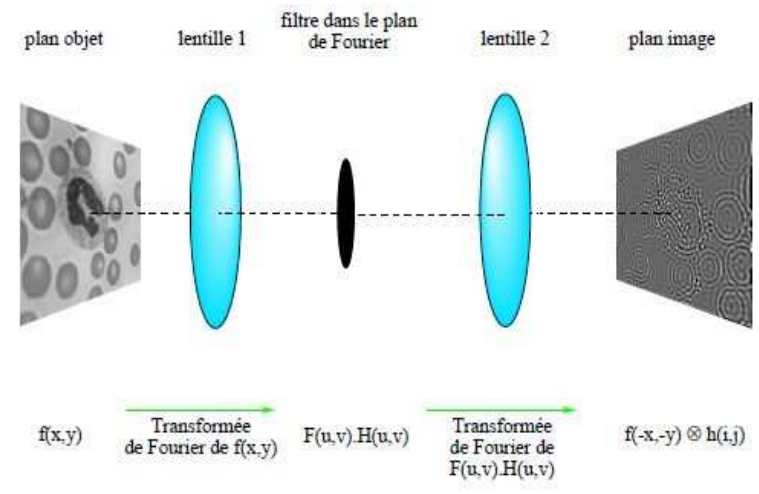

fig 13 : Filtrage passe-haut, strioscopie

Sur les fig 12 et 13 (ces schémas très explicites sont tirés de [5]) on précise l'opération de convolution correspondant au filtrage réalisé. La suppression de certaines fréquences au niveau de $\mathrm{PF}_{1}$ revient à multiplier la fonction des deux variables $p_{x}$ et $p_{y}$ (notées $u$ et $v$ sur les schémas) par une fonction bidimensionnelle contenant des 0 et des $1 \ldots$ aux bons endroits. La seconde transformée de Fourier (la diffraction à l'infini) s'effectue donc sur ce produit. Conformément au théorème bien connu, on obtient en $\mathrm{PF}_{2}$ l'image inversée convoluée avec la transformée du filtre.

\subsection{Filtrage des fréquences spatiales}

Les opérations de filtrage décrites en $\mathbf{3 . 2}$ se rapportent à des suppressions de fréquences relatives au spectre d'une image. On parle donc de filtrage des fréquences spatiales. Si dans l'image figure une trame parasite unidirectionnelle dont la fréquence est mesurable, la suppression de cette fréquence dans le spectre nettoiera théoriquement l'image de la trame. Cette retouche a bien sûr un prix puisque la fréquence supprimée, et l'ensemble des fréquences alentour, contiennent aussi des informations sur certaines des caractéristiques de l'image. En particulier, si la trame correspond à des oscillations rapides, la suppression des hautes fréquences correspondantes aura pour effet l'introduction d'oscillations parasites associées au phénomène de Gibbs [8], [9].

On voit fig 14 différents filtrages réalisés sur un spectre associé à une «grille» (fig 10) : un masque placé en $\mathrm{PF}_{1}$ ampute le spectre d'un ensemble de fréquences correspondant à une trame dans une direction donnée. Cette trame disparaît de l'image reconstituée en $\mathrm{PF}_{2}$ (en bas des deux figures fig 14). Dans le cas de la simulation numérique, notons que les «filtres » sont de simples « caches » ou « masques multiplicatifs ».
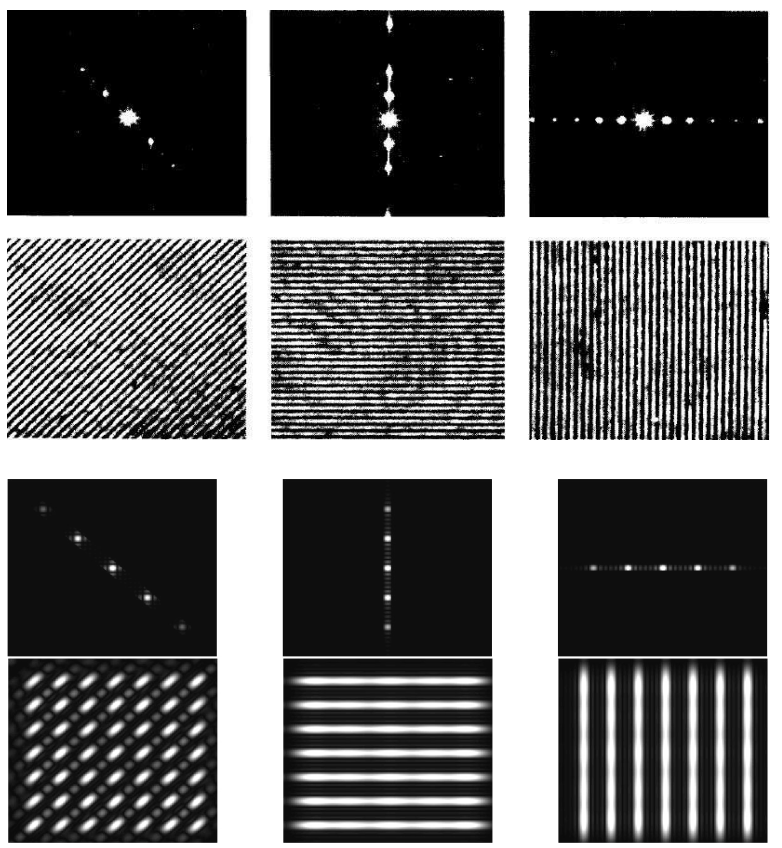

fig 14 : Filtrage des fréquences spatiales d'un « grille » : filtrage optique et simulation

\section{TRAITEMENT D'IMAGES}

\subsection{Introduction}

Les manipulations effectuées dans le montage d'Abbe indiquent clairement que l'on a pu modifier une image en intervenant sur son spectre. Ces opérations ont été effectuées dans l'espace physique « réel », et leurs conséquences ont été vues dans ce même espace. Dans ce contexte, on associe à la transformée de Fourier et à ses propriétés des processus physiques bien concrets.

On va, à présent, effectuer les mêmes opérations, ou presque, mais de façon numérique. On rentre alors dans le domaine du traitement d'images, où c'est en intervenant essentiellement au niveau de son spectre qu'une image est modifiée, retouchée, traitée. Dans notre étude, «filtrer» consiste simplement à placer un «masque multiplicatif» dans le plan des fréquences spatiales de l'image. La «reconstruction » après filtrage, correspond à la seconde partie du montage d'Abbe et à ce que l'on voit en $\mathrm{PF}_{2}$ (fig 11-13). 
Les deux grands effets auxquels on va être confronté sont les effets de repliement de spectre, ou d' "aliasing », dus à l'échantillonnage, et le phénomène de Gibbs dû à une troncature plus ou moins brutale du spectre. Tous deux introduiront des périodicités parasites, absentes des images avant reconstruction. $\mathrm{Si}$ ces deux effets sont omniprésents, on ne peut en théorie s'en affranchir complètement suite au «traitement » de l'image associé à un aller-retour entre l'espace réel et l'espace des fréquences spatiales, en particulier parce qu'un signal et son spectre ne peuvent être simultanément à support borné.

Pour notre étude, on va générer sous Matlab® une « image témoin » comprenant plusieurs motifs que l'on va d'abord analyser et traiter séparément. Ces motifs sont très simples pour être identifiables facilement d'un point de vue spectral, mais suffisamment variés pour que l'image soit intéressante et riche.

\subsection{Remarque importante}

Dans les simulations qui suivent, comme dans celles présentées plus haut, on a utilisé un logiciel de calcul où les images sont, par nature, «échantillonnées » et «fenêtrées ». De plus, leurs spectres sont obtenus par «transformée de Fourier discrète » (indépendamment de l'algorithme de calcul de FFT). Par conséquent, nous devrions tenir compte de ces propriétés intrinsèques et de leurs conséquences. Néanmoins, les situations dans lesquelles nous nous sommes placés nous permettent de les ignorer. Dans la suite, on fera donc comme si ces caractéristiques ne nous concernaient pas et on parlera de FFT pour désigner la transformée de Fourier au sens où l'on en parlait dans les parties $\mathbf{2}$ et $\mathbf{3}$.

\subsection{Un exemple simple : la suppression d'une trame}

Le motif fig 15 à gauche est composé de deux trames oscillantes orthogonales caractérisées par les fréquences spatiales 5 et $10 \mathrm{~m}^{-1}$ (unité arbitraire). La fonction de deux variables spatiales associée à ce motif est $S(x$, $y)=\sin (2 \pi 5 x) \cdot \sin (2 \pi 10 y)$. Son spectre obtenu par FFT est constitué de quatre raies très étroites associées aux deux fréquences dédoublées.
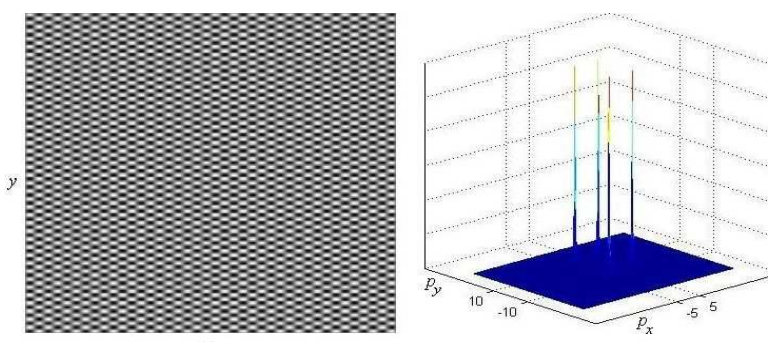

fig 15 : Sinusoïdes orthogonales et leur spectre

On vérifie à présent que la suppression d'un jeu de raies associé à une fréquence supprime bien l'oscillation correspondante dans l'image, exactement comme on l'a fait dans l'expérience d'Abbe. On crée pour cela un filtre simplement constitué d'un «cache » positionné au niveau des raies symétriques à supprimer.
Le filtre vaut 1 partout, sauf au niveau des raies où il vaut 0 . Une simple multiplication avec le spectre supprime les fréquences à enlever (fig 16).
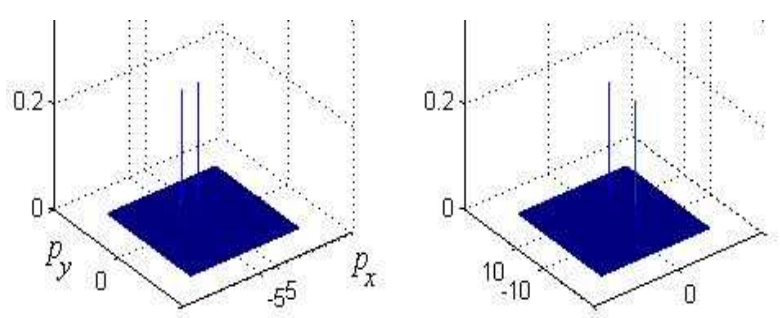

fig 16 : Suppression des fréquences $10 \mathrm{~m}^{-1}$ et $5 \mathrm{~m}^{-1}$

En effectuant la FFT des spectres filtrés fig 16, on obtient les figures initiales (inversées) desquelles l'oscillation correspondante a disparu (fig 17).
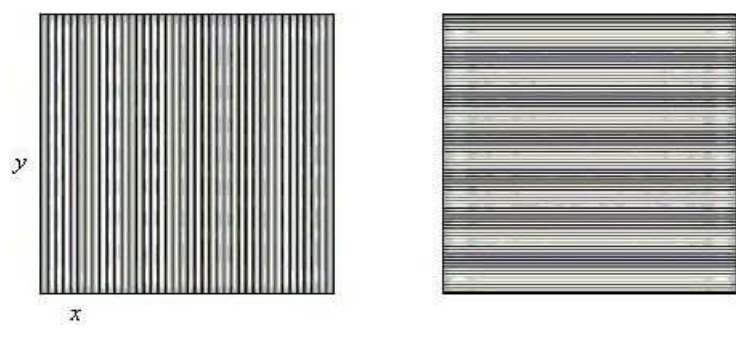

fig 17 : Oscillations à $10 \mathrm{~m}^{-1}$ et à $5 \mathrm{~m}^{-1}$ supprimées

\subsection{Traitement d'une image à plusieurs motifs}

\subsubsection{Description de l'image et de son spectre}

Pour commencer, on construit une «Image» dans laquelle figurent quatre motifs simples (fig 18 à gauche). Chaque motif a un spectre facile à identifier. Le motif en haut à gauche correspond à deux oscillations orthogonales à 5 et $10 \mathrm{~m}^{-1}$. Son spectre comprend quatre raies bien piquées à \pm 5 et \pm 10 . Le motif en bas à gauche est un ensemble de rayures horizontales. Son spectre se déduit de celui d'un ensemble de fentes. De même, les rayures obliques du motif en bas à droite conduisent à un spectre analogue au précédent, mais tourné d'un certain angle dans l'espace des fréquences spatiales. Enfin, le motif supérieur à droite ne comprend que des variations très progressives. Toute l'énergie de son spectre est donc concentrée dans les très basses fréquences spatiales, au centre du spectre.
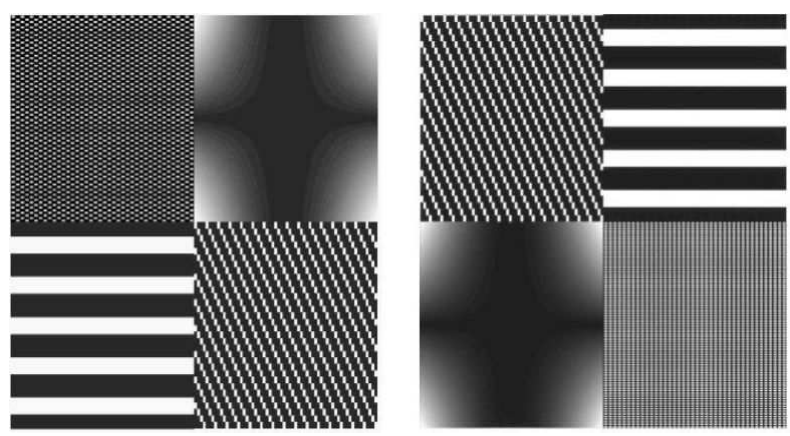

fig 18: L'Image et sa double FFT 
Comme on le constate, toutes les parties spectrales que l'on vient de décrire se retrouvent au sein du spectre global (en particulier les quatre raies à \pm 5 et \pm 10 ), bien que les rayures introduisent un enchevêtrement de raies. Remarquons que le spectre fig 19 ne donne aucune information sur les positions des motifs dans l'Image composite. La fig 18 à droite correspond à la FFT du spectre. Comme on s'y attendait, on obtient l'Image originelle, mais inversée par rapport à son centre.

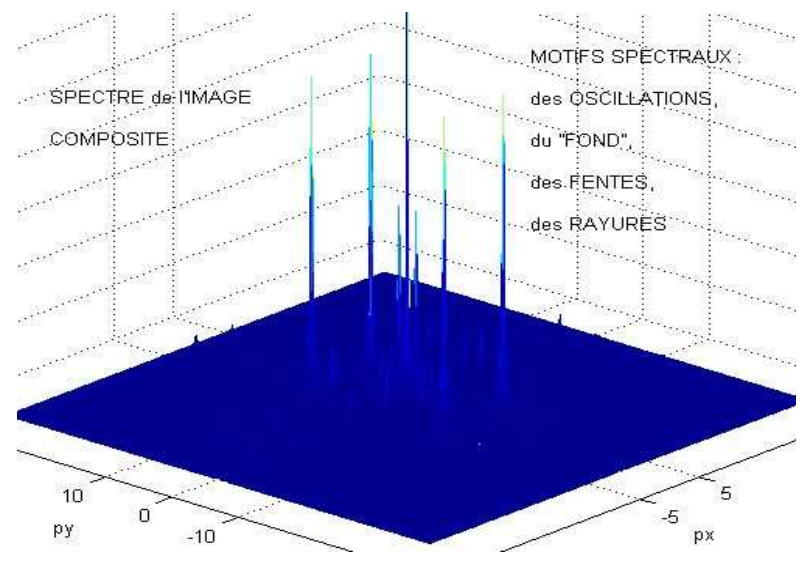

fig 19 : Spectre de l'Image composite

\subsubsection{Filtrage passe-bas et filtrage passe-haut}

$\mathrm{Au}$ vu du spectre fig 19, les parties du spectre correspondant à chaque motif seront différemment affectées selon le type de filtrage effectué. Après reconstitution, certains motifs seront donc modifiés ou dégradés plus que d'autres. A ce titre, la fig 20 (images agrandies) est très explicite. Un filtrage passe-bas adoucit les contours en introduisant des oscillations aux discontinuités, comme attendu (fig 20 à gauche). Les oscillations sinusoïdales en bas à droite (on n'oublie pas que la reconstitution restitue une image inversée) ont disparu: le filtrage a dû supprimer les quatre raies à \pm 5 et \pm 10 présentes dans le spectre fig 19. A droite de la fig 20, on observe la reconstruction de l'Image après un filtrage passe-haut. Les contours au niveau des rayures horizontales (en haut à droite) sont soulignés. Les oscillations sinusoïdales en bas à droite sont bien restituées. Par contre, la partie «lente » en bas à gauche a quasiment disparu.
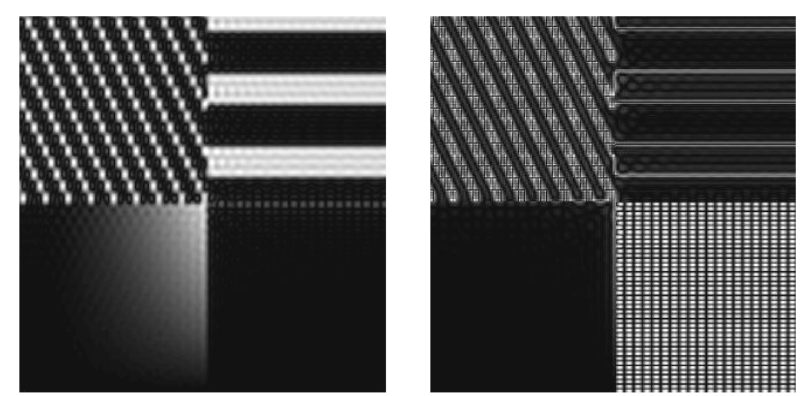

fig 20 : Reconstruction après filtrage $P B$ et $P H$

\subsubsection{Détramage}

Dans la même veine, on réalise le «détramage » d'une image parasitée par une oscillation horizontale, à une fréquence spatiale donnée : il s'agit de supprimer dans le spectre fig 21 (à droite) les deux pics bien visibles associés à la fréquence parasite.
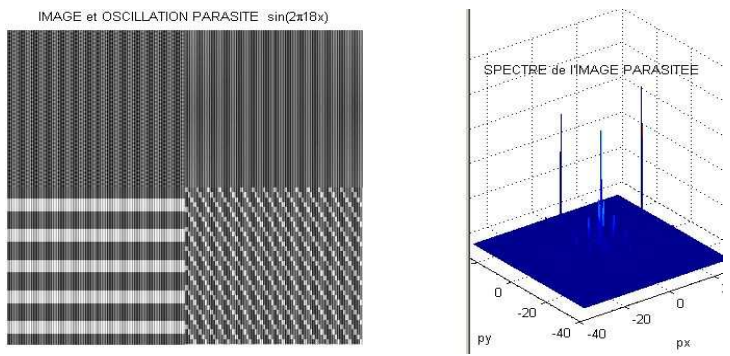

fig 21 : Image parasitée par une oscillation et spectre global

Un « cache multiplicatif » constitué de deux rectangles noirs (des «zéros ») placés aux bons endroits réalise cette amputation (fig 22 à gauche). Une FFT du spectre résultant conduit à l'image détramée, débarrassée de l'oscillation parasite. Il est clair qu'un filtrage aussi grossier entraîne, parallèlement, une perte d'information spectrale par une suppression de fréquences associées à l'image seule. On récupère donc une image détramée mais forcément «abimée » par des oscillations de Gibbs et des frontières légèrement érodées.

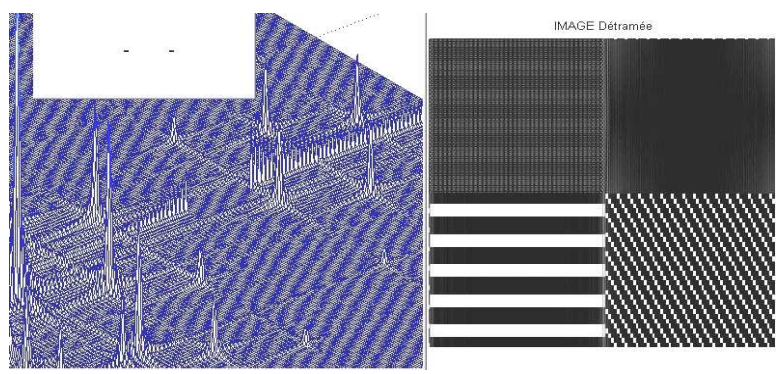

fig 22 : Suppression des pics associés à l'oscillation parasite et reconstruction : l'Image a été détramée... et un peu abimée

\subsubsection{Effets de l'échantillonnage}

Dans cette section, on va considérer les effets de 1' «échantillonnage » de 1'Image fig 18. Comme atten$\mathrm{du}$, on obtient, après échantillonnage, une duplication dans l'espace des fréquences spatiales du motif spectral de base de l'image non échantillonnée (fig 23).
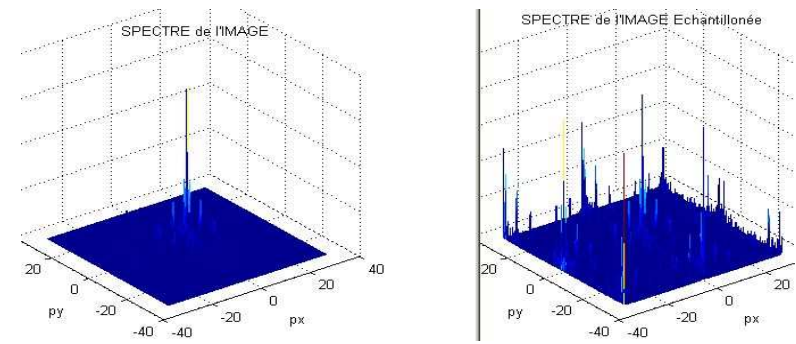

fig 23 : Spectre de l'Image avant et après échantillonnage 
Après sélection par filtrage - plus ou moins - passe-bas du motif spectral au centre (fig 24), puis reconstruction par FFT, on obtient les images (agrandies) présentées fig 25. La fig 25 a est obtenue par FFT du spectre filtré fig 24 à gauche. La fig 25 b est issue du filtrage présenté fig 24 à droite: on prend ici plus de hautes fréquences, risquant d'englober certaines fréquences des motifs dupliqués par l'échantillonnage. Par conséquent, le «repliement» sera bien présent, voire prédominant dans la reconstruction $b$, alors que le phénomène de Gibbs se manifestera, a priori, surtout dans celle en $a$.
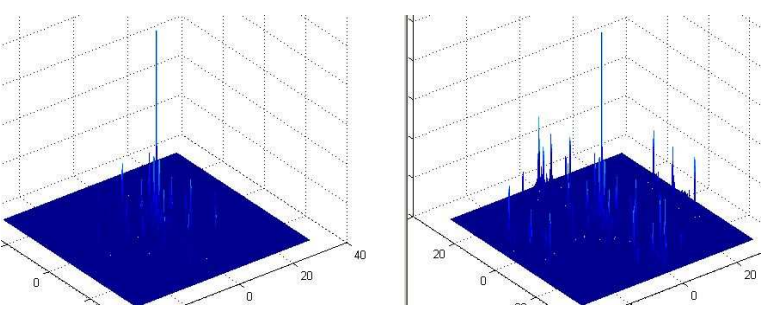

fig 24 : Deux filtrages du spectre de l'Image échantillonnée

Sur la coexistence de ces deux effets, les fig 25 a et $b$ sont particulièrement parlantes. En $b$, on remarque les phénomènes de repliement qui ont lieu, par exemple, au niveau des parties «rapides » de l'image (en haut à gauche au niveau des rayures obliques, en bas à droite au niveau des oscillations): des périodicités absentes de l'image originelle fig 18 y figurent. La fréquence d'échantillonnage aura donc été trop faible par rapport aux fréquences spatiales les plus hautes de l'image originelle. Parallèlement, le phénomène de Gibbs se manifeste aussi : des oscillations apparaissent au niveau des frontières dans l'image, au niveau des rayures en haut à droite, et en bas à gauche à la frontière de la zone «lente ». Dans la reconstruction fig $25 a$, le phénomène de Gibbs prédomine, a priori : dans le spectre filtré (fig 24 à gauche), il y a moins de fréquences parasites dues à la duplication, mais aussi peu de hautes fréquences.

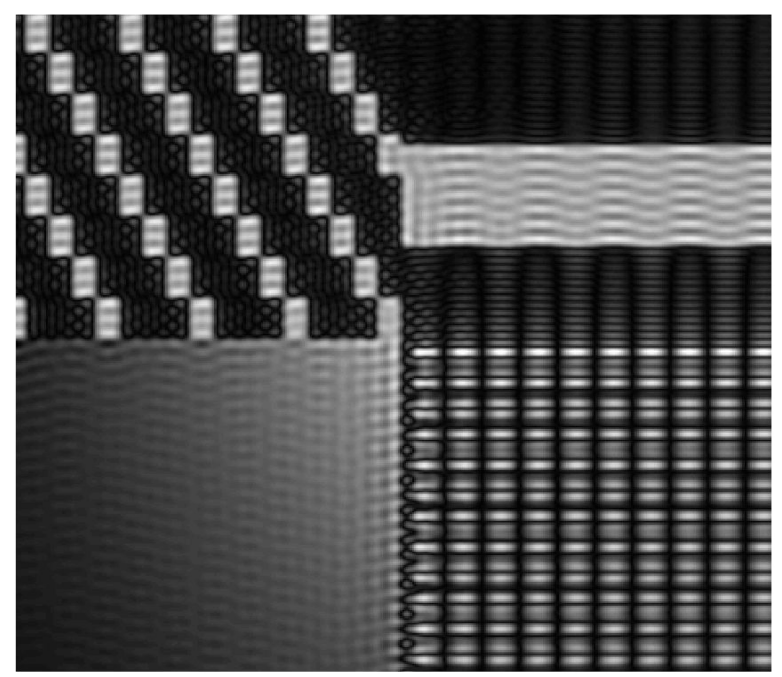

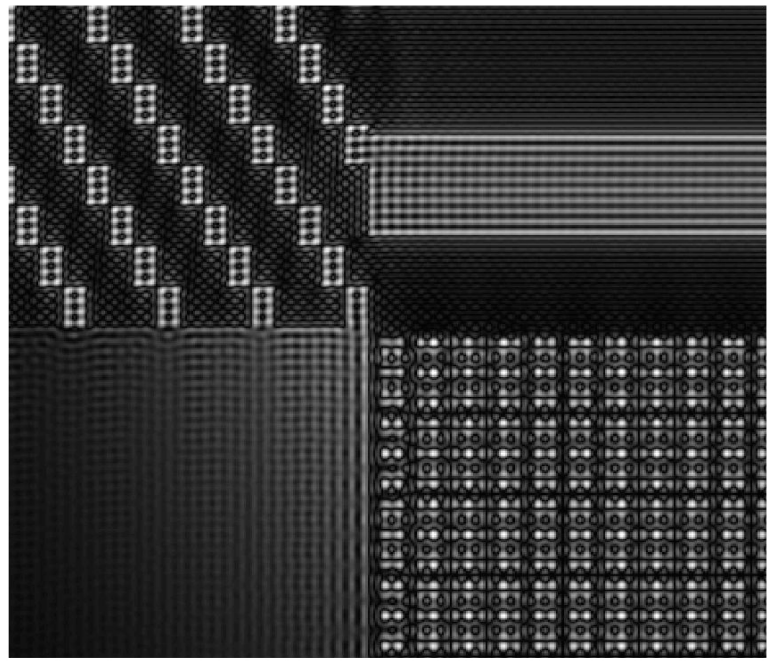

fig 25 a et $b$ : Reconstructions issues des spectres fig 24

\subsubsection{Reconnaissance et « corrélation »}

Pour terminer, on considère toujours l'Image fig 18 dans laquelle on a inséré divers motifs : une croix, un anneau et trois lettres F (fig 26).

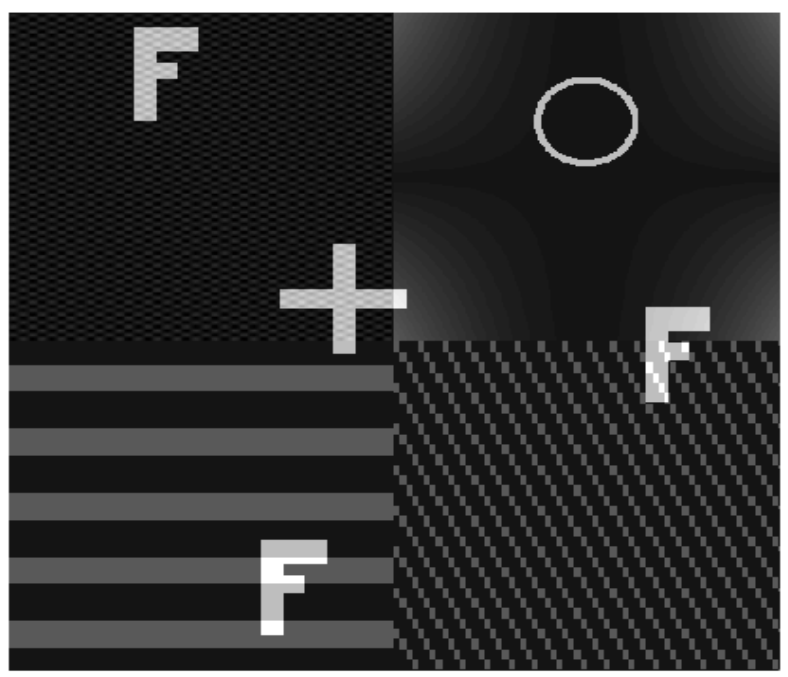

fig 26 : Notre Image contenant différents motifs

Il s'agit ici d'illustrer un résultat concernant la transformée de Fourier du «produit de corrélation », permettant la mise en évidence d'un «signal» noyé dans signal global. Dans le plan spectral de l'Image contenant les motifs «+ », « $\mathrm{O} »$, et « $\mathrm{F} »$, on place le conjugué du spectre du motif de référence que l'on désire repérer, en l'occurrence la lettre F majuscule (fig 27). 

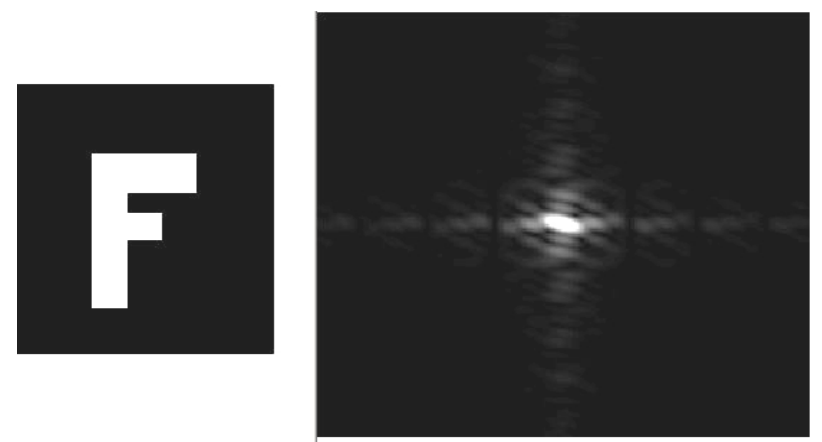

fig 27 : F majuscule et son spectre

Après avoir pris la FFT des deux spectres « combinés » (par un simple produit), on récupère l'image fig 28 dans laquelle se distinguent un certain nombre de pics : aux pics intenses correspond la présence du motif cherché, la lettre $\mathrm{F}$, dont on repère clairement les trois positions dans l'image. Un pic moins prononcé désigne la position de la croix au centre, dont la forme s'apparente à celle du F (un jeu de «fentes » orthogonales).

Enfin, une circonvolution très diffuse (en haut à droite) marque l'emplacement de l'anneau, très éloigné dans sa forme de celle du F.

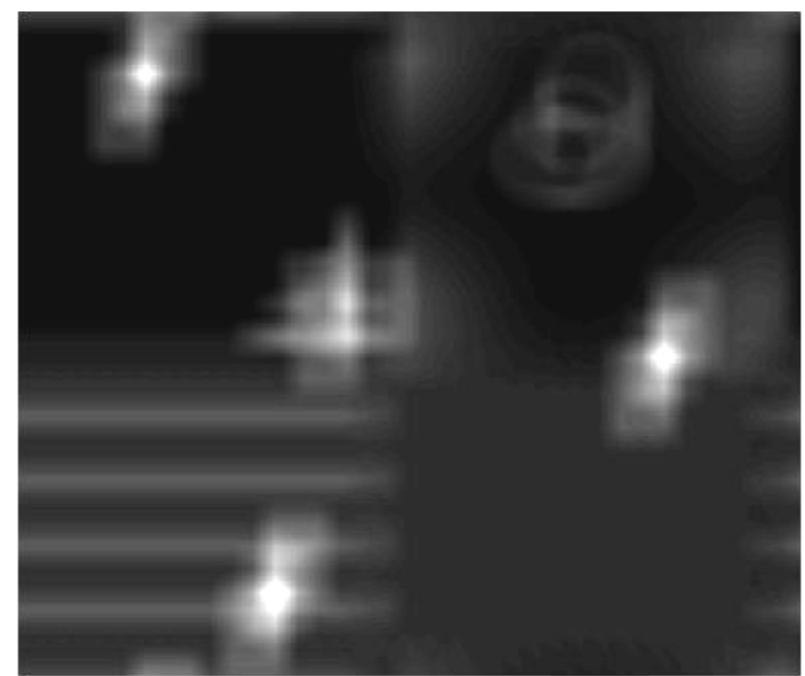

fig 28: Positions de la lettre F dans l'Image avec motifs

\section{CONCLUSION}

La compréhension et la maîtrise d'un ensemble de concepts nécessitent de pratiquer intensément le formalisme qui lui correspond. Dans les enseignements à consonance technologique, cette pratique porte rarement sur plusieurs domaines d'application. Pourtant, l'outil mathématique et les concepts qu'il exprime, existent en tant que tels et s'accommodent de tous les phénomènes physiques qui en sont comme les différentes réalisations. Les objectifs de cette présentation et du projet tuteuré proposé aux étudiants de DUT R\&T est précisément de cerner certains aspects d'un même outil - en l'occurrence la transformée de Fourier - en les mettant en parallèle dans deux contextes vus comme étran- gers l'un à l'autre. Des phénomènes appréhendés par les étudiants comme plus ou moins abstraits - invariance par décalage temporel, repliement de spectre, phénomène de Gibbs, effets de filtrage - apparaissent dans le contexte de l'Optique ondulatoire comme des manifestations bien visibles et localisées dans l'espace usuel. Ils acquièrent ainsi une portée et un statut qu'ils n'avaient pas dans l'étude exclusive et cloisonnée du traitement du signal, donnant aux étudiants et à leurs auditeurs une hauteur de vue qu'on peut vouloir dispenser dans nos enseignements.

Proposé depuis plusieurs années, ce projet tuteuré reste difficile, par les lectures et l'ouverture d'esprit qu'il réclame. Néanmoins, grâce à l'universalité de l'analyse de Fourier, les bénéfices que tous (étudiants et examinateurs) en retirent sont évidents : ouverture sur quelques grands thèmes de la Physique et recul vis-à-vis de la thématique particulière du traitement du signal.

Remerciements : je remercie les personnes chargées de l'organisation du Cetsis 2013 à Caen, pour toute l'attention et le soin apportés à la préparation et au bon déroulement du colloque. Je suis aussi reconnaissant au rapporteur de ma contribution, pour la lecture attentive et la présentation qu'il en a faite en début de séminaire.

\section{Bibliographie}

Les sources indiquées ci-après se rapportent aux différents thèmes abordés dans cet article : genèse des théories ondulatoires de la lumière [1], description détaillée des phénomènes ondulatoires de toute nature, en particulier ceux associés à l'Optique [2-4], phénomène de Gibbs [8-9] et traitement du signal [11]. Des URL sont indiquées pour les images et illustrations très explicites qu'elles renferment, [5] en particulier. Enfin, on pourra consulter dans l'annexe jointe les fichiers pdf des présentations faites au Cetsis 2013 et auxquelles cet article se rapporte: «poster» et version augmentée des «transparents ».

[1] André Chappert, « Histoire de l'optique ondulatoire. De Fresnel à Maxwell », Editions Belin, 2007.

[2] Franck S. Crawford Jr. « Cours de Physique de Berkeley. Volume 3 : Ondes », Librairie Armand Colin, 1972.

[3] Marcello Alonzo et Edward J. Finn, « Physique Générale.

Tome 2 : Champs et Ondes », InterÉditions, 1986.

[4] José-Philippe Pérez, « Optique. Fondements et applications », Editions Dunot, 2004.

[5] http://membres-timc.imag.fr/Yves.Usson/COURS/microfourier.pdf

[6] http://www.edu.upmc.fr/physique/joffrin 04001/chap5.1.pdf

[7] http://hal-sfo.ccsd.cnrs.fr/docs/00/29/25/69/PDF/chap6.pdf

[8] http://jf.burnol.free.fr/0506L312annexeGibbs.pdf

[9] http://www.math.ens.fr/culturemath/maths/pdf/analyse/shanno n.pdf

[10] http://sti.mermoz.free.fr/physique/TpPhyJTs2 fichiers/

[11] Philippe Courmontagne, «Ingénierie du signal, Théorie et pratique », Ellipses, Paris, 2005.

[12] Jean-Claude Pissondes, «Visualisation et mise en images des notions de base en traitement du signal : le traitement d'images et la transformée de Fourier à la lumière de la diffraction en Optique », Les cahiers pédagogiques des R\&T, Actes du 2ème colloque pédagogique, Kourou, Guyane, Novembre 2010, ISSN 2110-0330. 